\title{
Estudo experimental e computacional de um compressor de refrigeração operando sob condições controladas em uma câmara calorimétrica
}

\author{
Theoretical and experimental evaluation of a refrigeration compressor operating under \\ controlled conditions in a calorimetric chamber
}

\author{
I. C. A. Junior ${ }^{1}$; S. S. Pohlmann ${ }^{1}$; L. S. Silveira ${ }^{1}$; M. A. S. Rahn ${ }^{1}$; A. F. Porte ${ }^{1}$ \\ E. D. dos $\operatorname{Santos}^{2,3}$; L. A. Isoldi ${ }^{2,3}$; M. K. Rodrigues ${ }^{3 *}$ \\ ${ }^{1}$ Instituto Federal de Educação, Ciência e Tecnologia do Rio Grande do Sul (IFRS), 96.201-460, \\ Rio Grande-RS, Brasil \\ ${ }^{2}$ Escola de Engenharia (EE), Universidade Federal do Rio Grande (FURG),96.203-900,Rio Grande-RS, Brasil \\ ${ }^{3}$ Programa de Pós-Graduação em Modelagem Computacional (PPGMC), Universidade Federal do Rio Grande \\ (FURG) ,96.203-900,Rio Grande-RS, Brasil
}

*michel.professor@yahoo.com.br

(Recebido em 20 de outubro de 2014; aceito em 07 de março de 2015)

\begin{abstract}
A utilização de compressores com acionamento por frequência variável tem sido uma alternativa para a redução da energia elétrica em instalações de refrigeração.Neste trabalho, foram avaliados alguns parâmetros de desempenho de um compressor de refrigeração trabalhando com acionamento do motor elétrico sob frequências de $50 \mathrm{~Hz}$ e $60 \mathrm{~Hz}$, utilizando o fluido refrigerante R-404a. Dessa forma, foram realizadas medições em uma câmara calorimétrica submetida a temperatura constante de $32,2{ }^{\circ} \mathrm{C}$. As grandezas medidas foram: temperaturas de bulbo seco e úmido da câmara calorimétrica, velocidade do ar no evaporador, temperaturas de bulbo seco e umidade relativa do ar na área de face do evaporador e no insuflamento, pressões de sucção e descarga do compressor, tensão e corrente elétrica do motor de acionamento do compressor.Dessas medições foram obtidas a capacidade e a potência consumida pelo motor elétrico de acionamento do compressor, as quais foram utilizadas para determinação do coeficiente de performance e da eficiência isoentrópica do compressor. Além disso, foi realizda a modelagem matemática e a simulação computacional da capacidade de refrigeração do compressor, utilizando tanto os dados de desempenho fornecidos pelo fabricante, quanto aqueles provenientes das medições. Os resultados mostraram que o modelo obtido através das medições possui menores desvios do em relação ao desenvolvido através dos dados provenientes do fabricante para as condições estudadas. Além disso, os melhores desempenhos são alcançados para acionamento com frequência de $60 \mathrm{~Hz}$ em relação a de 50 $\mathrm{Hz}$.
\end{abstract}

Palavras-chave: Refrigeração por hidrocarbonestos, compressores, simulação computacional.

The use of compressors with a variable frequency drive has been an alternative to reduce energy in refrigeration. In this work, we evaluated some performance parameters of a refrigeration compressor working with electric motor drive frequencies under $50 \mathrm{~Hz}$ and $60 \mathrm{~Hz}$, using the refrigerant R-404a. Thus, measurements at a constant temperature of $32.2{ }^{\circ} \mathrm{C}$ were subjected held in an calorimeter chamber. The quantities measured were: temperature of dry and wet bulb calorimeter chamber, air velocity in the evaporator, the wet bulb temperature and relative humidity in the face area of the evaporator and insufflation, suction and discharge pressures of the compressor, voltage and electric current for the compressor motor. Capacity and power consumed by the electric motor of the compressor drive, which were used to determine the coefficient of performance and the isentropic compressor efficiency were measured. Furthermore, it was held mathematical modeling and computer simulation of the cooling capacity of the compressor, using both the performance data provided by the manufacturer, as those from the measurements. The results showed that the pattern obtained by the measurements has minor deviations in relation to the developed using data from the manufacturer to the conditions studied. Moreover, the best performers are achieved to drive with frequency of $60 \mathrm{~Hz}$ compared to $50 \mathrm{~Hz}$. Keywords: Refrigeration hidrocarbonestos, compressors, computer simulation. 


\section{INTRODUÇÃO}

$\mathrm{Na}$ atualidade muitos problemas envolvendo consumo energético na área de conservação de alimentos têm sido abordados, visando à produção e conservação de alimentos com menor custo agregado. Nesse sentido, aplicações no sentido de minimização de consumo de energia elétrica, otimização geométrica de materiais e equipamentos e a otimização termodinâmica de sistemas de refrigeração são tendências promissoras tanto na área de aplicação de refrigeração de pequeno e médio porte, quanto na indústria $[1,2,3]$.

Neste trabalho foi estudado um sistema de refrigeração de pequeno porte operando sob condições controladas em uma câmara calorimétrica. Os dados foram obtidos através de medições tanto no regime permanente, quanto no regime transiente, com o compressor frigorífico acionado por um motor elétrico operando nas frequências de $50 \mathrm{~Hz}$ e $60 \mathrm{~Hz}$. As grandezas medidas foram tensão e corrente do motor elétrico, temperaturas de bulbo seco e úmido do ambiente, pressões de sucção e descarga do compressor, temperaturas de bulbo seco e úmido da câmara estudada, e as velocidades do ar na entrada do evaporador.

Com esses dados foi possível determinar grandezas como: a capacidade, a potência consumida, coeficiente de performance $(C O P)$ e eficiência isoentrópica do compressor, bem como, o comportamento da temperatura e umidade relativa da câmara. Além disso, foi realizada a modelagem matemática e simulação computacional do funcionamento do compressor.

O ciclo de refrigeração por compressão de vapor é constituído pelo compressor, condensador, dispositivo de expansão,visor de líquido e evaporador. Além desses equipamentos, outros dispositivos acessórios podem ser incorporadas ao sistema a fim de garantir uma melhor qualidade na refrigeração do ambiente, um maior controle termodinâmico do processo, assim como para um maior auxílio de proteção [4].

Dessa forma, a busca por um melhor entendimento dos fenômenos de transferência de calor e massa através de modelagens matemáticas, simulações computacionais e ensaios experimentais vem sendo tema frequente de diversas pesquisas. Também no sentido de buscar melhor desempenho em sistemas de refrigeração, foram realizados estudos acerca de um novo tipo de compressor, avaliando, entre outros fatores, suas perdas por atrito, que é um dos causadores da geração de entropia no processo [5].

Dois conceitos importantes para o estudo da geração de entropia em processos de compressão são o de eficiência isoentrópica e o $C O P$. Segundo [6], eficiência isoentrópica envolve uma comparação entre o desempenho real de um equipamento e o desempenho que seria atingido em circunstâncias idealizadas para o mesmo estado inicial e a mesma pressão de saída. Esses fundamentos são expressos e modelados a seguir com base em [6, 7, 8].

Em um compressor, a forma da eficiência isoentrópica pode ser descrita segundo o diagrama de Mollier. O estado do fluído que entra no compressor e a pressão de descarga são constantes. Para desprezar a transferência de calor com a vizinhança e quaisquer efeitos de energia cinética e potencial, o trabalho da entrada por unidade de massa escoando através do compressor é dado por:

$$
-\frac{\dot{w}_{v c}}{\dot{m}}=h_{2}-h_{1}
$$

onde: $\dot{\mathrm{w}}_{\mathrm{vc}}$ é a potência $(\mathrm{kW}), \dot{m}$ é a vazão mássica $(\mathrm{kg} / \mathrm{s}), h_{2}$ e $h_{1}$ são as entalpias $(\mathrm{kJ} / \mathrm{kg})$ na descarga e sucção do compressor, respectivamente.

Uma vez que o estado inicial é fixo, a entalpia específica $h_{1}$ é conhecida. Assim sendo, o valor da potência de entrada dependa da entalpia específica da saída $h_{2}$. Essa expressão mostra que a magnitude da potência de entrada decresce à medida que $h_{2}$ decresce. A potência mínima da entrada corresponde ao menor valor permitido para a entalpia específica na saída do compressor. O menor valor permitido da entalpia no estado de saída seria alcançado em uma compressão isoentrópica a partir do estado especificado na entrada até a pressão de saída especificada. A potência mínima da entrada é fornecida por: 


$$
-\frac{\dot{w}_{v c}}{\dot{m}}=h_{2 s}-h_{1}
$$

Em uma compressão real, tem-se que $h_{2}>h_{2 s}$, consequentemente mais potência do que a mínima seria necessário. Essa diferença pode ser medida pela eficiência isoentrópica do compressor definida por:

$$
\eta_{c}=\left[\frac{-\left(\frac{\dot{w}_{v c}}{\dot{m}}\right)_{S}}{-\left(\frac{\dot{w}_{v c}}{\dot{m}}\right)}\right]
$$

onde: $\eta_{c}$ é a eficiência isoentrópica do compressor.

Já o desempenho do sistema foi determinado através do COP (coefficient of performance) expresso por:

$$
C O P=\frac{\dot{q}}{\dot{w}}
$$

onde: $\dot{q}$ é a potência de resfriamento ou capacidade do sistema $(\mathrm{kW})$ e $\dot{w}$ é a entrada de potência no compressor $(\mathrm{kW})$. Para [6] o COP é definido como a relação entre o efeito desejado e a entrada de potência necessária em um sistema de refrigeração, enquanto que para [8] o mesmo é definido como a capacidade de resfriamento do sistema e a entrada de potência no compressor.

\section{MATERIAL E MÉTODOS}

Nesta seção é apresentada a instalação de refrigeração analisada neste trabalho. Sendo assim, foi realizada a descrição do estudo experimental e a abordagem computacional do sistema em estudo. Também foi apresentada a modelagem matemática utilizada para a aplicação da metodologia empregada neste trabalho.

\subsection{Instalação de refrigeração}

O estudo experimental foi realizado em uma câmara calorimétrica estabilizada localizada em um laboratório termofluidico no IFRS Campus Rio Grande. Neste procedimento foram observadas as recomendações contidas em [6], mantendo-se a temperatura ambiente em $32,2^{\circ} \mathrm{C}$ (Figura 1). Também foram seguidas as normas técnicas citadas em [9, 10, 11, 12, 13, 14]. Os dados experimentais utilizados neste estudo foram oriundos de 60 medições realizadas em cada condição de acionamento do motor elétrico $(50$ e $60 \mathrm{~Hz})$.

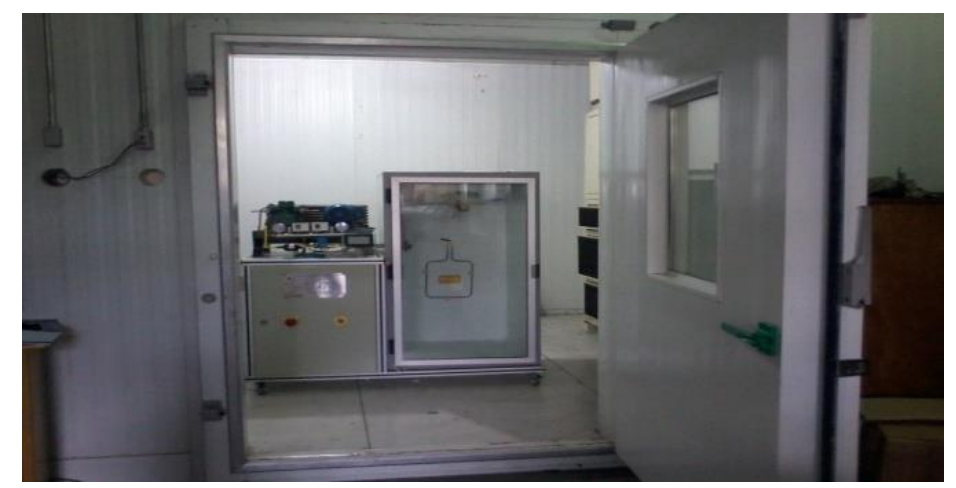

Figura 1: Sistema de refrigeração estudado.

Esse sistema trata-se de uma instalação básica (sistema comercial de pequeno porte) de refrigeração por meio de compressão de vapor, operando com o fluido refrigerante R-404a. Esse sistema permitiu a variação da frequência de acionamento, sendo possível a medição das 
seguintes variáveis: temperatura de bulbo seco de insuflamento, temperatura de bulbo seco de retorno, umidade relativa de retorno e de insuflamento, tensão e corrente do motor elétrico, temperaturas de bulbo seco e úmido do ambiente, pressões de sucção e descarga do compressor, temperaturas de bulbo seco e úmido da câmara estudada, e as velocidades do ar na entrada do evaporador, a fim de calcular as propriedades do sistema.

\subsection{Modelagem matemática e computacional}

A modelagem matemática utilizada neste trabalho segue os princípios da termodinâmica e de aplicações em sistemas de refrigeração $[4,6,7]$. A modelagem computacional foi realizada através do software EES (Engineering Equation Solver). O EES é frequentemente utilizado na solução de problemas de engenharia na área de termodinâmica e transferência do calor. Esse software é constituído de um solver robusto capaz de atender de forma rápida e segura simulações computacionais implementadas [15].

Primeiramente, foi determinada a capacidade do sistema dada pela expressão:

$$
\dot{q}=\rho V A\left(h_{r}-h_{i}\right)
$$

onde: $\rho$ é a massa específica do $\operatorname{ar}\left(\mathrm{kg} / \mathrm{m}^{3}\right), V$ é a velocidade do $\operatorname{ar}(\mathrm{m} / \mathrm{s}), A$ é a área de face do evaporador $\left(\mathrm{m}^{2}\right), h_{r}$ é entalpia de retorno $(\mathrm{kJ} / \mathrm{kg})$ e $h_{i}$ é a entalpia de insuflamento $(\mathrm{kJ} / \mathrm{kg})$.

As grandezas envolvidas para determinação da capacidade do sistema foram determinadas através das medições de velocidade, temperaturas de bulbo seco e úmido, bem como das dimensões do evaporador. A velocidade do ar e as condições psicrométricas do ar de retorno foram medidas junto à área de face do evaporador seguindo as recomendações da norma [10]. As propriedades termodinâmicas do ar foram determinadas com o auxílio do software EES.

Em seguida foi determinada a relação de compressão $\left(R_{c}\right)$, dada por:

$$
R_{c}=\frac{P_{c}}{P_{e}}
$$

onde: $P_{c}$ é a pressão de condensação $\left(\mathrm{N} / \mathrm{m}^{2}\right)$ e $P_{e}$ é a pressão de evaporação $\left(\mathrm{N} / \mathrm{m}^{2}\right)$.

Já as temperaturas de evaporação $\left(t_{e v}\right)$ e de condensação $\left(t_{c}\right)$, ambas em $\left({ }^{\circ} \mathrm{C}\right)$, foram determinadas por meio das pressões de saturação $P_{e}$ e $P_{c}$, respectivamente.

A vazão mássica do fluido refrigerante foi determinada por:

$$
\dot{m}=\frac{\dot{q}}{h_{v}-h_{l}}
$$

onde: $h_{v}$ é a entalpia do fluido refrigerante na saída do evaporador $(\mathrm{kJ} / \mathrm{kg})$ e $h_{l}$ é a entalpia do fluido refrigerante no estado líquido na entrada do dispositivo de expansão.

A potência real de compressão $\left(\dot{W}_{\text {real }}\right)$, em $(\mathrm{W})$, foi assim determinada:

$$
\dot{W}_{\text {real }}=U I \sqrt{3} \cos \varphi
$$

onde: $U$ é a tensão de acionamento do motor elétrico (V), $I$ é a corrente elétrica (A), $\cos \varphi$ é o fator de potência. Como simplificação, considerou-se o conjunto motor compressor como único componente.

Reescrevendo-se a Equação (3) utilizando as Equações (2) e (8) tem-se:

$$
\eta_{c}=\frac{\dot{m}\left(h_{2 s}-h_{1}\right)}{U I \sqrt{3} \cos \varphi}
$$


A capacidade frigorífica, a vazão em massa, a eficiência e a potência requerida pelo sistema em um dado regime de operação pode ser calculada através de polinômios de terceiro grau dado por:

$$
\begin{aligned}
& X=C_{1}+C_{2}\left(t_{e v}\right)+C_{3}\left(t_{c}\right)+C_{4}\left(t_{e v}{ }^{2}\right)+C_{5}\left(t_{e v} t_{c}\right)+C_{6}\left(t_{c}{ }^{2}\right)+C_{7}\left(t_{e v}{ }^{3}\right)+C_{8}\left(t_{c} t_{e v}{ }^{2}\right)+ \\
& C_{9}\left(t_{e v} t_{c}{ }^{2}\right)+C_{10}\left(t_{c}{ }^{3}\right)
\end{aligned}
$$

O polinômio representado pela Equação (10) expressa as variáveis em função das temperaturas correspondentes ao regime operacional e seus coeficientes $C_{i}$, onde: $i=$ $(1,2,3, \ldots, 10)$ devem ser determinados através de dados fornecidos pelos fabricantes do equipamento.Neste trabalho foram definidos os polinômios referentes à capacidade frigorífica, cujos coeficientes foram definidos através dos dados fornecidos pelo fabricante e através das medições feitas.

\section{RESULTADOS E DISCUSSÃO}

O estudo realizado no sistema de pequeno porte comercial com frequências de $50 \mathrm{~Hz}$ e $60 \mathrm{~Hz}$ oportunizou a determinação da capacidade, da potência do sistema e também COP do compressor por meio das medições realizadas.

A equação representativa da capacidade depende das temperaturas de evaporação e de condensação determinada através dos dados fornecidos pelo fabricante. A mesma serviu para comparar o comportamento esperado do sistema com aquele obtido através dos dados provenientes das medições.

As Figuras 2 (a) e 2 (b) mostram o comportamento das temperaturas de retorno $\left(T_{1}\right)$ e de insuflamento $\left(T_{2}\right)$ do ar da câmara, bem como da temperatura de evaporação $\left(T_{e v}\right)$ do sistema para medição em regime transiente com o motor elétrico acionado por uma frequência de $50 \mathrm{~Hz}$ e $60 \mathrm{~Hz}$, respectivamente. O comportamento em ambos os casos é semelhante, com uma redução mais rápida da temperatura nos minutos iniciais de funcionamento, tendendo a um valor aproximadamente constante após 15 min. (minutos) de operação. Uma tendência bastante clara também é a diferença de temperatura entre o ar de retorno e de insuflamento e entre estas e a temperatura de evaporação, que se mostra praticamente constante nos ensaios realizados a partir do momento em que o sistema passa a operar em regime permanente. Para acionamento do motor elétrico com frequência de $50 \mathrm{~Hz}$ a diferença entre a temperatura da câmara e de evaporação estabiliza em valores próximos a $9,5^{\circ} \mathrm{C}$, enquanto que para frequência de $60 \mathrm{~Hz}$ esta diferença se mantém em torno de $12,5^{\circ} \mathrm{C}$. Isso se deve ao fato que com a frequência de $50 \mathrm{~Hz} \mathrm{o}$ deslocamento volumétrico do compressor é de aproximadamente $83,3 \%$ do deslocamento realizado com uma frequência de acionamento de $60 \mathrm{~Hz}$.

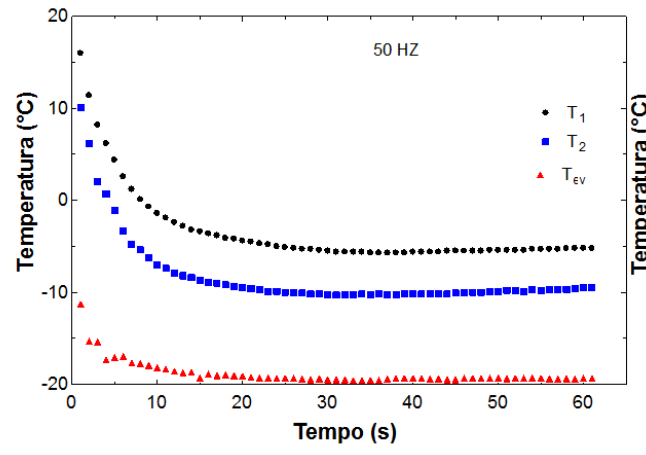

(a)

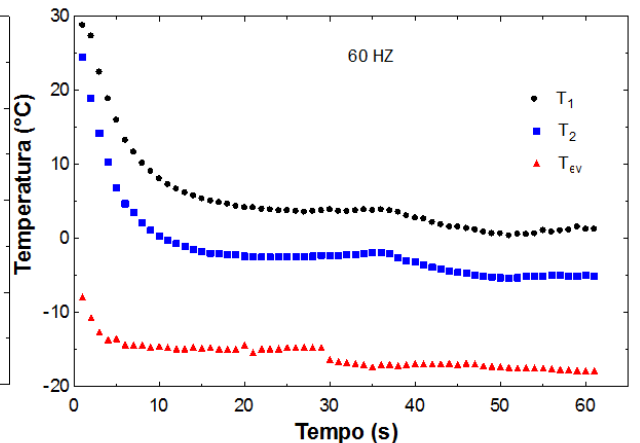

(b)

Figura 2: Variação das temperaturas do ar da câmara e de evaporação para acionamento com $50 \mathrm{~Hz}$ (a) $e$ variação das temperaturas do ar da câmara e de evaporação para acionamento com $60 \mathrm{~Hz}(b)$. 
Através da modelagem matemática e da simulação computacional, foi possível traçar uma comparação entre as capacidades esperadas pela simulação e aquelas descritas pelo fabricante. A Figura 3 (a) mostra a curva perfeitamente correlaciona no que se refere aos dados preditos e os determinados pelo fabricante, bem como os dados calculados. Para tais condições, a correlação $\left(R^{2}\right)$ determinada foi igual a $100 \%$. Na Figura 3 (b) esta tendência é evidenciada, visto que os dados de capacidade fornecidos pelo fabricante são muito próximos daqueles obtidos pela simulação para temperaturas de condensação de $30^{\circ} \mathrm{C}, 40^{\circ} \mathrm{C}$ e $45^{\circ} \mathrm{C}$. As temperaturas de evaporação consideradas variam de $-45^{\circ} \mathrm{C}$ à $-10^{\circ} \mathrm{C}$. Para temperaturas de evaporação de $-45^{\circ} \mathrm{C}$ as capacidades são próximas de $0,15 \mathrm{~kW}$, aumentando na medida em que estas temperaturas também aumentam, alcançando valores próximos de $1,0 \mathrm{~kW}$ para temperaturas de evaporação de $-10^{\circ} \mathrm{C}$ e de condensação de $30^{\circ} \mathrm{C}$.

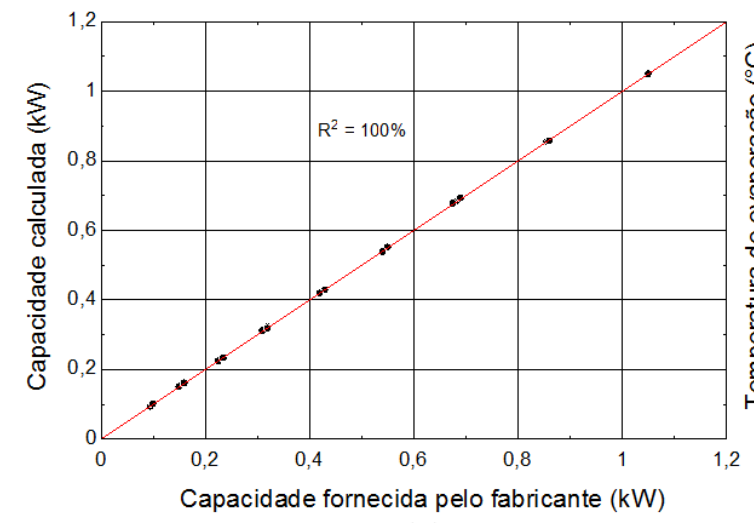

(a)

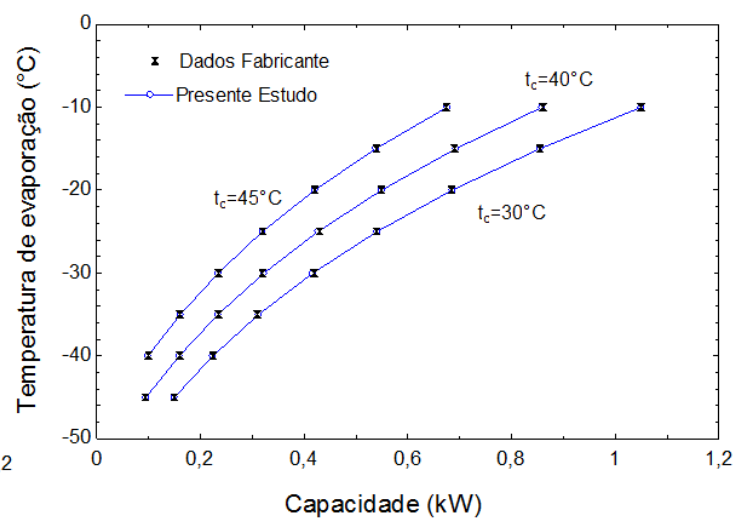

(b)

Figura 3: Capacidade calculada versus capacidade fornecida pelo fabricante (a) e capacidade em função da temperatura de evaporação $(b)$.

As Figuras 4 (a) e 4 (b) apresentam uma comparação entre a capacidade medida e a capacidade calculada pelo modelo matemático utilizado para as temperaturas de evaporação e condensação ensaiadas, para $50 \mathrm{~Hz}$ e $60 \mathrm{~Hz}$, respectivamente. Nos dois casos as maiores diferenças encontradas foram inferiores a $0,15 \mathrm{~kW}$, mostrando uma boa convergência do modelo com os dados experimentais para a faixa de medição estudada. Considerando-se a propagação de incertezas do processo de medição, resultados ainda mais próximos poderão ser encontrados, conforme pode ser visto nas Figuras 5 (a) e 5 (b).

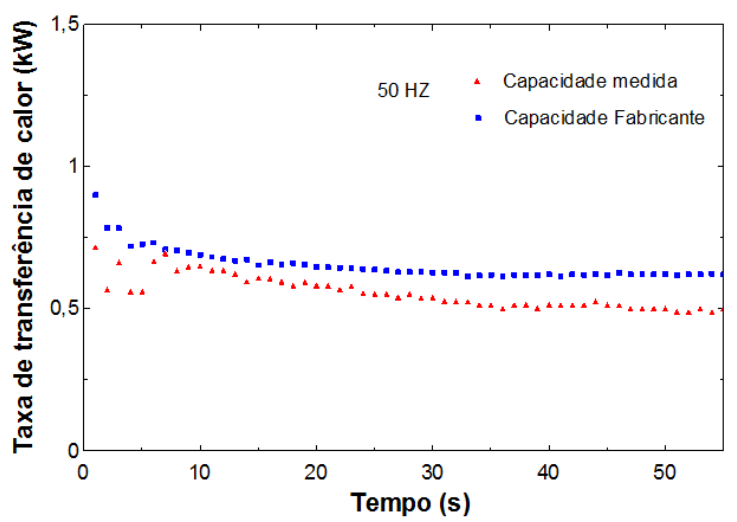

(a)

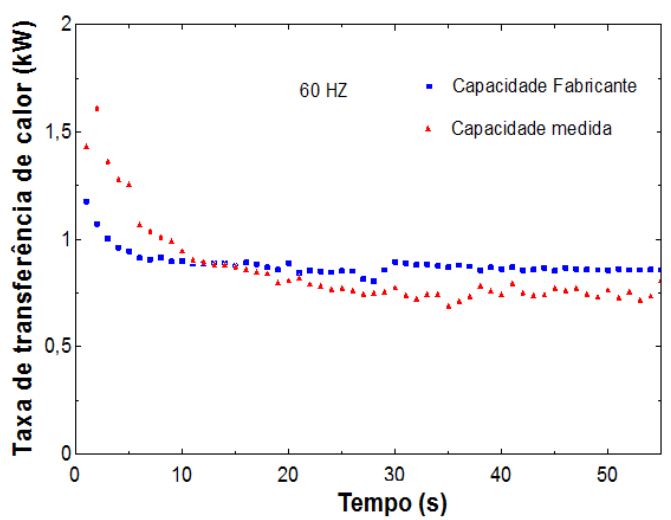

(b)

Figura 4: Taxa de transferência de calor para $50 \mathrm{~Hz}$ (a) e taxa de transferência de calor para $60 \mathrm{~Hz}$ (b).

Outra possibilidade é realizar a modelagem matemática a partir dos dados experimentais. Os resultados deste procedimento podem ser verificados na Figura 5, onde se pode observar a curva significativamente bem ajustada aos resultados experimentais. A correlação $\left(R^{2}\right)$ obtida para tal condição foi de $97,83 \%$. 


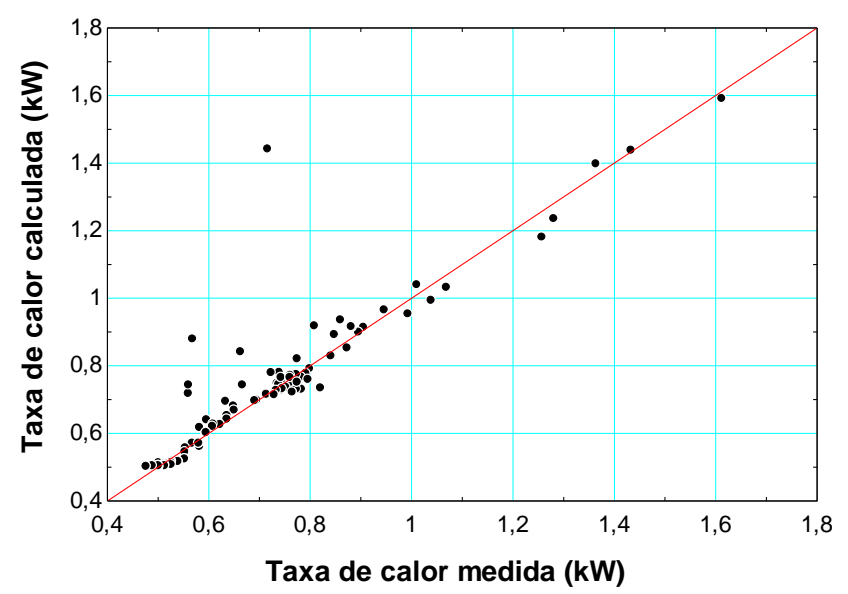

Figura 5: Correlação entre dados experimentais e simulados computacionalmente.

As Figuras 6 (a) e 6 (b) mostram os valores da taxa de transferência de calor $(\dot{q})$ obtida através das grandezas medidas, simulada através da modelagem feita com os dados fornecidos pelo fabricante e simulada através da modelagem feita com os dados oriundos das medições. A Figura 6 (a) apresenta os valores para condição de ensaio de $50 \mathrm{~Hz}$. Nesta condição, observa-se que a modelagem feita através dos dados provenientes das medições apresenta muito boa convergência com aqueles calculados diretamente das grandezas medidas. Com tempos de ensaio menores que $5 \mathrm{~s}$, algumas diferenças significativas foram encontradas, sendo bastante atenuadas para nos instantes seguintes. Em poucos casos os desvios foram em torno de 5\%, sendo os demais inferiores a este valor. Comparando-se os valores calculados através das grandezas medidas, foram observadas diferenças, em alguns casos, próximas de $20 \%$.

Para os ensaios realizados com frequências de $60 \mathrm{~Hz}$ (Figura 6 (b) foram encontrados desvios menores entre os calculados pelas medições e através da modelagem matemática, mostrando novamente uma boa convergência. No que diz respeito à convergência entre dados medidos e modelagem através dos dados do fabricante, novamente foram verificados maiores desvios, alcançando, em alguns casos, valores próximos de $15 \%$ para dados medidos a partir de $5 \mathrm{~s}$. Em ambos os casos (Figuras 6 (a) e 6 (b)) estes maiores desvios no início das medições deve-se ao pouco tempo para estabilização dos instrumentos de medição e da câmara frigorífica ensaiada, uma vez que o sistema ainda encontrava-se em regime transiente.

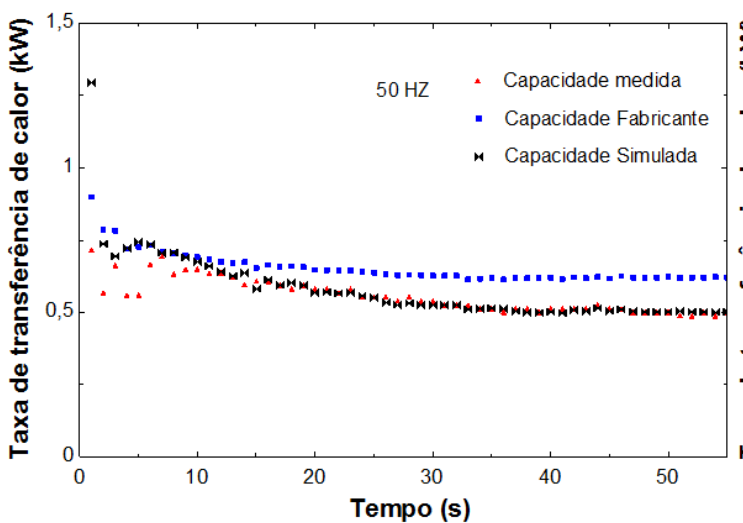

(a)

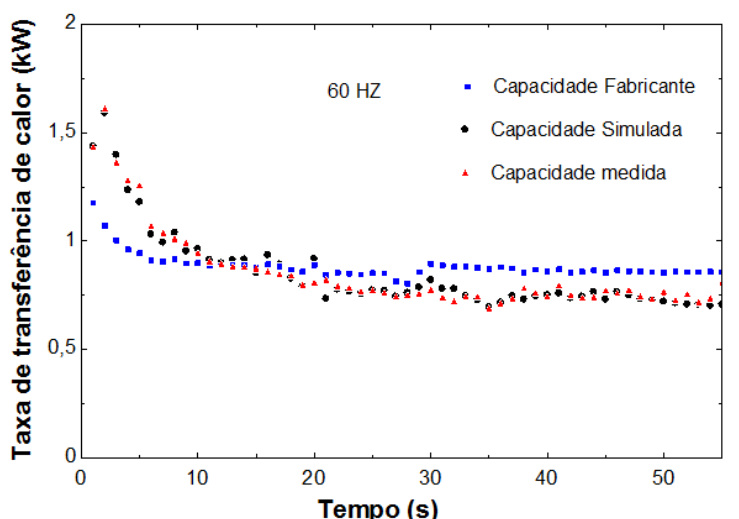

(b)

Figura 6: Capacidade do sistema para $50 \mathrm{~Hz}$ (a) e capacidade do sistema para $60 \mathrm{~Hz}$ (b).

A Eficiência isoentrópica do compressor estudado também foi verificada, sendo seus valores apresentados nas Figuras 7 (a) e 7 (b), para acionamento com frequências de $50 \mathrm{~Hz}$ e $60 \mathrm{~Hz}$, respectivamente. Pode-se observar que em ambos os casos a relação de compressão possui grande influência sobre a eficiência isoentrópica. Para o caso de acionamento com $50 \mathrm{~Hz}$, na 
medida em que a relação de compressão aumenta de 4,8 para 5,5 a eficiência se reduz de aproximadamente 0,19 para 0,14, apresentando alguns poucos dados dispersos. Quando o acionamento foi feito com $60 \mathrm{~Hz}$, a eficiência reduziu de aproximadamente 4,5 (para relação de compressão próxima de 4,0) para aproximadamente 0,27 quando a relação de compressão se aproxima de 5,0. Notadamente, o acionamento efetuado com $60 \mathrm{~Hz}$ apresenta melhores valores de eficiência isoentrópica quando comparado com o acionamento feito com frequência de 50 $\mathrm{Hz}$.

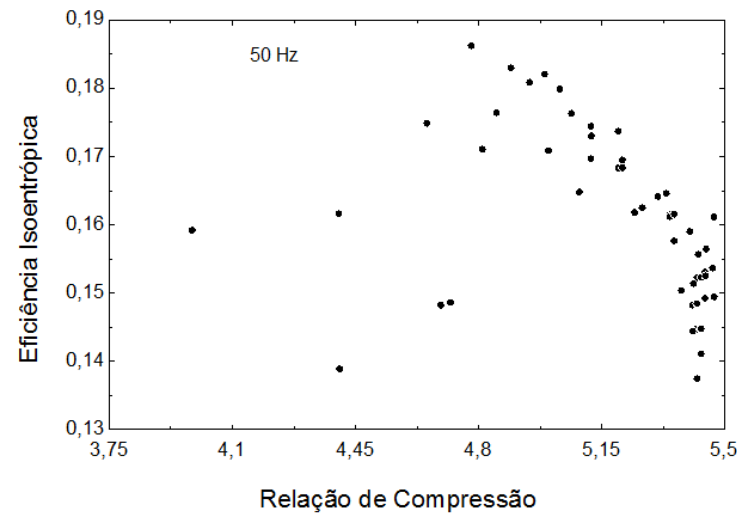

(a)

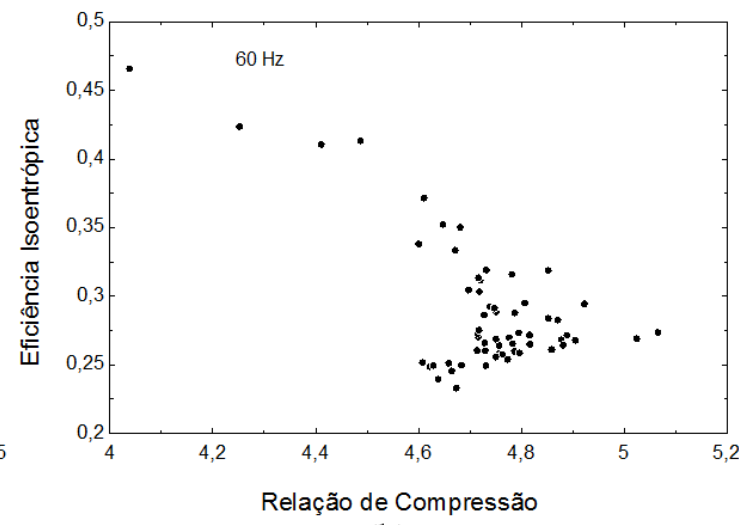

(b)

Figura 7: Eficiência isoentrópica do compressor em função da relação de compressão para $50 \mathrm{~Hz}$ (a) e $60 \mathrm{~Hz}(b)$.

Tendo em vista esta diferença de comportamento em relação a eficiência isoentrópica do compressor para acionamento com diferentes frequências, cabe também investigar $o$ comportamento do COP do mesmo quando submetido às mesmas condições de acionamento anteriores. Através das Figuras 8 (a) e 8 (b) pode-se também verificar que o COP segue a mesma tendência que a eficiência isoentrópica quando a relação de compressão varia, apresentando maiores valores de performance para $60 \mathrm{~Hz}$ do que para $50 \mathrm{~Hz}$.

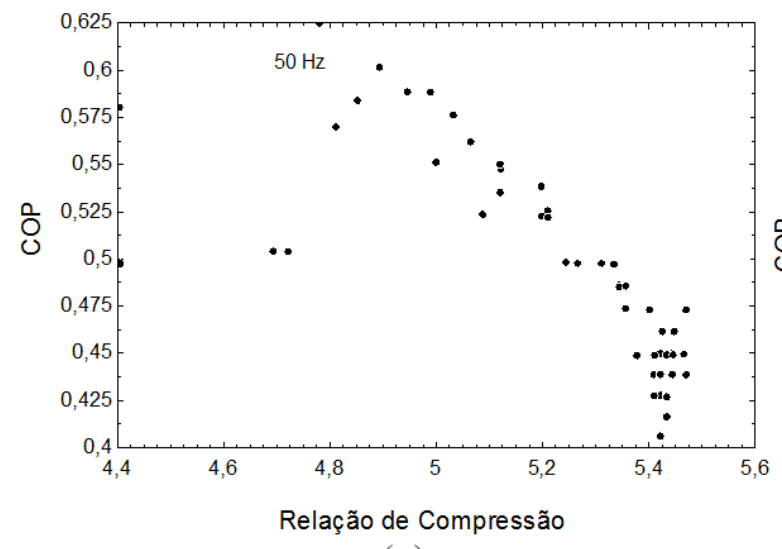

(a)

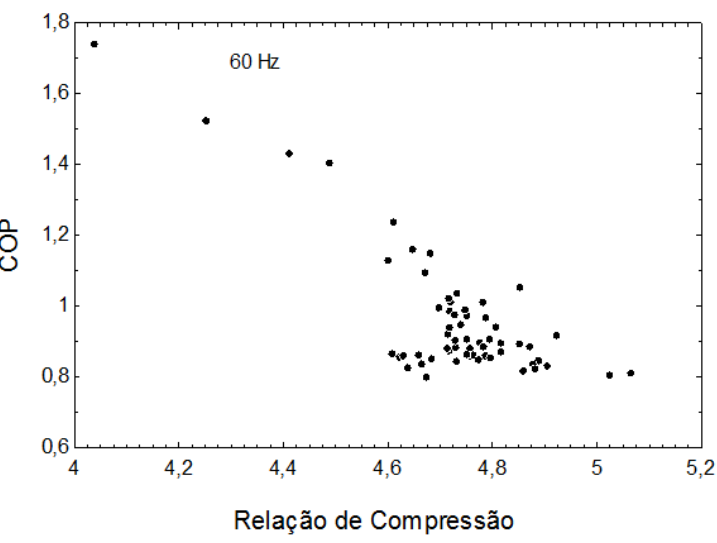

(b)

Figura 8: COP do compressor em função da relação de compressão para $50 \mathrm{~Hz}($ a) e $60 \mathrm{~Hz}(b)$.

\section{CONCLUSÃO}

Este trabalho contou com um estudo experimental e computacional de um compressor de refrigeração do tipo aberto operando em um ambiente controlado. As medições efetuadas demonstraram que a diferença entre as temperaturas de evaporação e internas da câmara onde se encontra o evaporador permaneceram praticamente constantes durante os testes. A modelagem 
matemática realizada a partir dos dados fornecidos pelo fabricante mostrou ser exata em relação a estes dados, entretanto apresentou algum desvio em relação aos dados experimentais.

A modelagem matemática realizada por meio dos dados experimentais apresentou menores desvios em relação à modelagem feita com os dados do fabricante. No entanto, novos ensaios com temperaturas de condensação de $30^{\circ} \mathrm{C}, 40^{\circ} \mathrm{C}$ e $45^{\circ} \mathrm{C}$ se fazem necessários a fim de comparar os dois modelos matemáticos obtidos.

Quanto ao desempenho do compressor considerando-se tanto o COP, quanto a eficiência isoentrópica. As avaliações realizadas com o motor elétrico acionado com frequência de $60 \mathrm{~Hz}$ mostraram melhores resultados em relação àquelas com $50 \mathrm{~Hz}$.

\section{AGRADECIMENTOS}

Os autores agradecem ao CNPq pelo apoio.

\section{REFERÊNCIA BIBLIOGRÁFICAS}

1. Bejan A, Tsatsaronis G, Moran M. Thermal design and optimization. John Wiley \& Sons, Inc.; 1996. New York, 542 p.

2. Ding G. Recent developments in simulation techniques for vapour-compression refrigeration systems. International Journal of Refrigeration. 2007 ;30:1119-1133.

3. Ferrari F. Otimização termodinâmica de sistemas de refrigeração solar, Dissertação de Mestrado, Programa de Pós-Graduação em Engenharia Mecânica (PPGEM). Universidade Federal do Paraná (UFPR); 2005.

4. Silva J de C, Silva ACG. Refrigeração e climatização para técnicos e engenheiros. Rio de Janeiro, Ciência Moderna; 2007. 346 p.

5. Teh YL, Ooi KT. Theoretical study of a novel refrigeration compressor - part I: design of the revolving vane (RV) compressor and its frictional losses. Int. J. Refrigeration. 2009;33:1092-1102.

6. Moran MJ, Shapiro HN. Fundamentals of Engineering Thermodynamics. Fifth Edition. John Willey and Sons Ltd; 2006.

7. Çengel A, Boles A. Termodinâmica. 5. ed. São Paulo: McGraw-Hill; 2006. 740 p.

8. Wang B, Shi W, Han L, Li X. Optimization of refrigeration system with gas-injected scroll compressor. Int. J. Refrigeration. 2009;32;1544-1554.

9. ISO 917: Testing of refrigerant compressors. 2 ed. Genève (Switzerland); 1989.

10. NBR 10142. Associação Brasileira de Normas Técnicas. Condicionador de ar tipo compacto. Ensaios de aceitação em fábrica. Método de ensaio. Rio de Janeiro, RJ: ABNT; 1987. 22 p.

11. NBR 15371. Associação Brasileira de Normas Técnicas. Evaporadores tipo circulação forçada para refrigeração. Especificação de desempenho e identificação; 2006

12. NBR 15372. Associação Brasileira de Normas Técnicas. Resfriadores de ar para refrigeração. Métodos de Ensaio; 2006.

13. NBR 15627-1. Associação Brasileira de Normas Técnicas. Condensadores a ar remotos para refrigeração. Parte 1: Especificação, requisitos de desempenho e identificação; 2008.

14. NBR 15826. Associação Brasileira de Normas Técnicas. Compressores para refrigeração- Métodos de ensaio; 2010.

15. Klein A, Alvarado L. EES - Engineering Equation Solver; F-Chart Software, Middleton, WI. 1995. 